\title{
Autoevaluación y Toma de Decisiones para Mejorar la Competencia Oral en Educación Secundaria
}

\author{
Self-Assessment and Decision-Making for the Improvement of Oral Competence in \\ Secondary Education
}

\author{
Marta Gràcia ${ }^{1}$, Jesús M. Alvarado ${ }^{2}$ y Silvia Nieva ${ }^{3}$
}

\begin{abstract}
Resumen
La incorporación en los centros educativos de las Tecnologías de la Información y la Comunicación (TIC) supone una gran oportunidad para la formación de los docentes, así como para el desarrollo de competencias por parte del alumnado. En la presente investigación se utiliza un Sistema Inteligente de Asistencia Pedagógica (SIAP) diseñado para formar al docente, ayudándole a diseñar sus clases, autoevaluando el progreso respecto a distintos hitos para lograr clases más participativas que fomenten la competencia oral del alumnado. El SIAP fue utilizado durante diez semanas en un diseño experimental con grupo control: 188 estudiantes de Educación Secundaria, 84 grupo intervención y 104 grupo comparación. Los indicadores que incluye mostraron cómo, a medida que se lograban los hitos docentes, mejoraba la competencia oral del alumnado. Complementariamente, en un cuestionario se observó un cambio positivo en el grupo de intervención en el autoconcepto del alumnado sobre distintas habilidades comunicativas.
\end{abstract}

Palabras clave: autoevaluación, toma de decisiones, sistema inteligente de asistencia pedagógica, competencia comunicativa oral, Educación Secundaria, formación del profesorado

\begin{abstract}
The incorporation in the teaching of Information and Communication Technologies (ICTs) that are evolving towards proposals that incorporate Artificial Intelligence (AI) represents a great opportunity for more meaningful learning. There are few AI initiatives aimed at teachers. This research uses an Intelligent Pedagogical Assistance System (SIAP) designed to help the teacher to design their classes, self-evaluating progress in different items to achieve more participatory classes that promote oral competence. The SIAP was used for ten weeks in an experimental design with a control group: 188 Secondary Education students: 84 intervention group and 104 comparison group. The indicators included showed how, as the teaching items were achieved, oral competence improved. In addition, in an independent questionnaire a positive change in self-concept about different communication skills was observed in the intervention group.
\end{abstract}

Keywords: self-assessment, decision-making, intelligent pedagogical assistance system, oral communicative competence, Secondary Education, teacher training

Agradecimientos: Proyecto I+D+i del Ministerio de Ciencia, Innovación y Universidades: Aplicación Digital de Autoevaluación y toma de decisiones para la mejora de la Competencia Oral y el Rendimiento Académico en Educación Secundaria (ADACORA) (2020-2023). Proyecto coordinado entre la Universidad de Barcelona (UB) (PID2019-105177GB-C21) (IP: Marta Gràcia) y la Universidad Complutense de Madrid (UCM) (PID2019-105177GB-C22) (IP: Jesús M. Alvarado)

${ }^{1}$ Doctora en Psicología. Profesora Titular, Facultad de Psicología, Universidad de Barcelona. Barcelona, España. Passeig de la Vall d’Hebrón, 171. Tel.: (+34) 933125832. Correo: mgraciag@ub.edu

${ }^{2}$ Doctor en Psicología. Profesor Titular. Facultad de Psicología Universidad Complutense de Madrid. Campus de Somosaguas, 28223 Pozuelo de Alarcón, Madrid. España. Tel.: (+34) 913943115. Correo: jmalvara@ucm.es

${ }^{3}$ Doctora en Psicología. Logopeda. Profesora Ayudante Doctora. Facultad de Psicología Universidad Complutense de Madrid. Campus de Somosaguas, 28223 Pozuelo de Alarcón, Madrid. España. Tel.: (+34) 913943115. Correo: silnieva@ucm.es

Revista Iberoamericana de Diagnóstico y Evaluación - e Avaliação Psicológica. RIDEP · No62 · Vol.1 · 83-99 2022

ISSN: 1135-3848 print /2183-6051online 


\section{Introducción}

Presentamos el análisis de una experiencia de formación de profesorado de Educación Secundaria en el que se implementó un Sistema Inteligente de Asistencia Pedagógica (SIAP) flexible y abierto, la Escala de Valoración de la Lengua Oral en contexto Escolar - Sistema de Soporte a la toma de Decisiones, EVALOE-SSD (Gràcia et al., 2020a, 2020b). Con su implementación se pretende un cambio innovador de foco a través del uso de una herramienta que facilita a cada docente reflexionar sobre su práctica, evaluarla, tomar decisiones, y mejorar sus clases.

La formación del profesorado no siempre presta la suficiente atención a la forma de enseñar (Girvan et al., 2016; Smagorinsky et al., 2015). En muchos centros educativos se sigue trabajando desde una perspectiva en la que el conocimiento se entiende como algo específico del docente, no fomentando el diálogo entre profesor y alumnos, en general dirigido por el primero, y otorgando escaso valor al diálogo entre alumnado (Wells, 2009; Reznitskaya, 2012; Millard \& Menzies, 2016). Sin embargo, introducir una práctica reflexiva puede servir como punto de conexión entre el conocimiento del docente y sus acciones, permitiéndole evaluar su propia práctica, reflexionar sobre los nuevos patrones de comportamiento en el proceso de cambio, y experimentar con ellos (Berson et al., 2015; Sedova, 2017). Esta permanente reflexión es necesaria para poner en práctica una adecuada autorregulación orientada a objetivos. Distintas investigaciones (Capa-Aydin et al., 2009; Chatzistamatiou et al., 2014; Persico et al., 2015), han evidenciado que existe una relación entre el grado en la utilización de estrategias de autorregulación por parte del profesorado y la sensación de eficacia (Capa-Aydin et al., 2009). Por su parte, en el trabajo de Chatzistamatiou et al. (2014) se muestra que las estrategias de autorregulación, tanto para su propia enseñanza como para mejorar la del alumnado, eran predecibles por sus creencias de autoeficacia en la enseñanza de matemáticas, el valor que atribuyen a las matemáticas y su compromiso emocional con su profesión.

En una línea similar, en el estudio de van Beek et al. (2014) se analizan las actividades propuestas (externas, compartidas e internas) por los docentes para ayudar a los estudiantes a autorregular su proceso de aprendizaje, resaltando que los profesores combinaron la instrucción directa (regulación externa) con actividades de aprendizaje estimulantes, de sondeo (regulación compartida) y autorreguladas (regulación interna) para regular, estimular y mejorar las actividades de aprendizaje de los estudiantes.

En las últimas décadas se han desarrollado diversas herramientas tecnológicas para proporcionar retroalimentación al profesorado sobre sus propias prácticas y estimular la reflexión (Romano \& Schwartz, 2005). A pesar de que los medios digitales son un recurso fundamental en formación inicial y desarrollo docente (Fainholc et al., 2013; Hughes et al., 2016; Paredes et al., 2015), gran parte de la investigación se ha centrado en las competencias para ser usuarios de tecnología (Koh, 2019; Uerz et al., 2018) y alcanzar la competencia digital profesional (Gewerc \& Montero, 2015; Instefjord \& Munthe, 2017). No obstante, en algunos estudios se han evaluado usos de la tecnología que ayudan a los profesores a reflexionar sobre sus prácticas docentes y a introducir mejoras (Könings \& Gijselaers, 2015; Mosley-Wetzel et al., 2017; Prieto et al., 2017; Reid et al., 2015; Romano \& Schwartz, 2005), habiéndose observado que en el manejo de los recursos digitales, el uso de Sistemas de Soporte de Decisiones (SSD) ha mostrado ser de ayuda para definir y aclarar la toma de decisiones de los docentes y sus consecuencias (Arnott \& Pervan, 2016; Compton, 2009; Walsh, 2003). Desde esta línea de investigación surge la EVALOE-SSD como una herramienta para ayudar al docente en la programación de sus actividades en el aula realizando un seguimiento constante de su progreso en la mejora de la comunicación en el aula.

EVALOE-SSD se ha elaborado para conseguir que las clases se conviertan en espacios de comunicación, diálogo, reflexión y evaluación sobre el propio proceso de aprendizaje y enseñanza de todos los contenidos del currículum, por parte tanto del alumnado como de los docentes, con el fin último de contribuir al desarrollo de todas las competencias (Gràcia et al., 2020a, 2020b). La primera versión de la 
EVALOE-SSD fue usada como instrumento de formación de profesorado, de autoevaluación y de toma de decisiones, de manera que las aulas se transformaron progresivamente en contextos en los que el foco eran los alumnos, conversando entre ellos, dialogando, preguntando, reflexionando, y gestionando la conversación gracias al modelado de las maestras. Los resultados en Educación Primaria han mostrado cómo la implementación de este instrumento contribuye a mejorar la competencia oral del alumnado (Gràcia et al., 2020a, 2020b).

El marco conceptual que subyace a la EVALOE-SSD se sitúa en una perspectiva constructivista de orientación sociocultural (Coll, 2010) desde la que se entiende la interacción entre profesor y alumno -la enseñanza y el aprendizajecomo un proceso de ayuda y guía por parte del profesor a la co-construcción del conocimiento por parte del alumnado (van Schaik et al., 2019) en base a los contenidos curriculares, que promueve el desarrollo de todas las competencias (Marope et al., 2017). Una de ellas es la competencia comunicativa, que se contempla como un contenido a enseñar y aprender, a la vez que como un instrumento para aprender los contenidos vinculados a las otras áreas de desarrollo/aprendizaje.

La EVALOE-SSD forma parte de la investigación educativa en la que, en los últimos años, ha incrementado el interés en cómo los estudiantes participan en las discusiones en clase. Es un interés que se enmarca en la teoría sociocultural, representada especialmente por Vygotsky (1978). Desde esta perspectiva, la participación de los estudiantes en estas discusiones juega un papel muy relevante (Sedlacek \& Sedova, 2016; Sedova et al., 2019). Así, Molinari y Mameli (2013) afirman que el grado de participación de los estudiantes puede ser considerado una medida de la calidad general del discurso en clase, y en un nivel más general, de la calidad de la educación, objetivos para los que la EVALOE-SSD puede contribuir de forma decisiva. Esta contribución parte de que se trata de una herramienta tecnológica diseñada para ser flexible y abierta para ayudar a los profesores a reflexionar sobre su práctica, evaluarla, tomar decisiones y mejorar sus propias habilidades $\mathrm{y}$ estrategias.
El interés del presente estudio parte de la exploración del potencial de EVALOE-SSD en Educación Secundaria. Se trata de explorar mediante una experiencia de formación del profesorado, el uso, acogida y valoración de esta herramienta por parte de los docentes en esta etapa educativa para promover el desarrollo de la competencia oral en el alumnado adolescente. Partimos de las siguientes preguntas de investigación: 1) ¿La EVALOE-SSD como recurso de aprendizaje permite mejorar las prácticas docentes?; 2) ¿El uso de la EVALOESSD mejora la competencia oral del alumnado a juicio del profesorado?; 3) ¿La autopercepción de competencia oral del alumnado cambia a lo largo del periodo de formación del profesorado?; 4) ¿En qué dimensiones se detectan los avances más importantes?

\section{Método}

\section{Instrumentos}

La versión de la EVALOE-SSD que se implementa en este estudio fue elaborada en base a un instrumento anterior, la EVALOE, una escala de observación (Gràcia et al., 2015a, 2015b) que ha sido validada como instrumento de reflexión docente (Gràcia et al., 2021b) en Educación Primaria. Concretamente, un psicólogo de la educación y un logopeda participaron en un proceso de confiabilidad entre evaluadores para la primera versión de la escala de observación. Se realizaron ocho observaciones en el aula en una escuela. Los dos evaluadores evaluaron de forma independiente la sesión observada en cada clase al mismo tiempo. La fiabilidad entre evaluadores para la EVALOE fue alta, con un coeficiente kappa de Cohen $\kappa=.87$ (Cerda \& Villarroel, 2008). Los ítems con baja fiabilidad fueron revisados para garantizar que no hubiera problemas con la redacción, y ningún ítem fue modificado o eliminado después del proceso de evaluación de la fiabilidad.

Como herramienta SIAP, la EVALOE-SSD incluye recursos de naturaleza variada: un tutorial con información sobre su objetivo, su uso, el tipo de recursos que incluye, los pasos que se recomienda ir siguiendo, entre otros. Contiene también una pantalla para que el docente describa 
la clase que se propone evaluar destacando los elementos que considera más relevantes, lo cual ya supone un primer nivel de reflexión sobre su práctica.

La herramienta proporciona ejemplos y guías diversas para la elaboración de esta descripción. El principal recurso es un conjunto de 30 ítems con tres opciones de respuesta cualitativa (emoji de cara verde, naranja y roja) que propone al docente evaluar de manera sistemática pero flexible sus clases en relación con cinco dimensiones en función de su contenido: 1) diseño instruccional (por ejemplo, «el alumnado autoevalúa su competencia comunicativa»), 2) gestión de la conversación por parte del docente (por ejemplo, «la disposición del mobiliario y de los participantes se ajusta a las características de la actividad»; 3) gestión de la conversación por parte del alumnado (por ejemplo, «adoptamos un formato de interacción en red durante las actividades de conversación y discusión», 4) estrategias comunicativas docentes (por ejemplo, «enseño a sintetizar y/o extraer conclusiones»; y 5) funciones del alumnado (por ejemplo, «el alumnado utiliza fórmulas de interacción social»).

Existen ítems de diferente grado de complejidad (tres niveles), así como ítems vinculados exclusivamente a las acciones del docente, del alumnado, e ítems que incluyen a ambos. El docente puede consultar una serie de ayudas-recursos en dos tipos de formato: 1) preguntas generadoras de reflexión sobre el ítem o acción en concreto (por ejemplo: «¿Cómo puedo contribuir a que consideren a sus compañeros/as como personas que pueden aportar nuevas e interesantes informaciones, que los ayudan a resolver sus dudas, a aprender, a desarrollar competencias de tipo diverso?»); 2) vídeo corto de una situación real de clase que ilustra la acción/ítem con una explicación.

Una vez asignado un color a cada ítem que aparece en el cuestionario en función de las características específicas de la clase, el docente puede consultar un cuadro resumen con todos los ítems y las valoraciones que ha asignado a cada uno. A continuación, la herramienta inteligente le propone que tome una decisión. Concretamente aparecen 6 tarjetas vinculadas a 6 ítems, a los que ha asignado un color naranja o rojo, y le propone que elija 3 , y que los intente introducir en sus siguientes clases. El docente toma esta decisión de manera libre y reflexiva a partir de su experiencia previa con el asistente pedagógico, de sus expectativas y de las condiciones concretas de su asignatura. Finalmente, el docente tiene un espacio para escribir las anotaciones que considere que le pueden ser útiles para revisar en otro momento, discutir con otros docentes o con el alumnado.

Otro instrumento utilizado en este trabajo es un Cuestionario de Autopercepción de Competencia Oral dirigido al alumnado. Este instrumento consiste en un conjunto de preguntas agrupadas en 4 dimensiones (gestión de interacción, prosodia, cohesión textual y estrategias argumentativas), que fueron evaluadas en trabajos anteriores del equipo (Gràcia et al., 2020c, 2021a) para valorar el progreso de la competencia oral de alumnado universitario a partir de la introducción por parte del profesorado de intervenciones orientadas a situar la conversación y la discusión como elementos clave de aprendizaje en las aulas universitarias. Su objetivo es detectar el grado en el que la introducción de nuevas metodologías centradas en la conversación, en la evaluación y en la reflexión sobre la competencia oral por parte del profesorado ha impactado en la autopercepción del alumnado en cuanto a su propia competencia oral. Esta investigación servirá para presentar las primeras evidencias de validez de este instrumento.

Un tercer instrumento utilizado es un Cuestionario de Satisfacción formado por preguntas abiertas y cerradas para explorar la valoración de la implementación del SIAP por parte del profesorado. Esta investigación servirá también para presentar las primeras evidencias de validez de este otro instrumento.

\section{Participantes}

La muestra se seleccionó a través de un muestreo no probabilístico por conveniencia. Participaron docentes y alumnado de centros públicos pertenecientes a dos Comunidades Autónomas (regiones) de España: Cataluña y la Comunidad Autónoma de Madrid. Participaron seis docentes que impartían las asignaturas de Castellano, Catalán, Alemán, Inglés, Francés y Ciencias Sociales, 84 alumnos de primer y tercer 
Tabla 1. Categorías y códigos para analizar los datos cualitativos (descripciones de las clases) de los docentes de los grupos de intervención

\begin{tabular}{|c|c|}
\hline Categorías & Códigos \\
\hline 1. Ayudas EVALOE -SSD & $\begin{array}{l}\text { 1.1. Ayudas de EVALOE-SSD más usadas } \\
\text { 1.2. Referencia a la utilidad de las ayudas } \\
\text { 1.3. Ayudas escritas de EVALOE-SSD } \\
\text { 1.4. Uso de la ayuda en formato vídeo } \\
\text { 1.5. Uso de la ayuda en formato imagen }\end{array}$ \\
\hline $\begin{array}{l}\text { 2. Valoración del uso de EVALOE- } \\
\text { SSD }\end{array}$ & $\begin{array}{l}\text { 2.1. Lenguaje empleado en la redacción de los ítems } \\
\text { 2.2. Modo como se responde cuestionario (seguido/fragmentado) } \\
\text { 2.3. Interpretación de los símbolos/iconos } \\
\text { 2.4. Importancia del idioma } \\
\text { 2.5. Estrategias usadas durante la familiarización con la App } \\
\text { 2.6. Tiempo de dedicación y reflexión sobre la app } \\
\text { 2.7. Acceso a la app } \\
\text { 2.8. Número de ítems } \\
\text { 2.9. Organización del contenido } \\
\text { 2.10. Tres niveles de respuesta (verde, amarillo y rojo) } \\
\text { 2.11. Toma de decisiones de los tres objetivos tomados }\end{array}$ \\
\hline $\begin{array}{l}\text { 3. Razones de la dificultad de aplicar } \\
\text { ítems (decisiones) en clase }\end{array}$ & $\begin{array}{l}\text { 3.1. Número de lenguas que están aprendiendo los niños } \\
\text { 3.2. Habilidades lingüísticas/comunicativas de los niños } \\
\text { 3.3. Nivel de complejidad de los ítems } \\
\text { 3.4. Cantidad de ítems }\end{array}$ \\
\hline $\begin{array}{l}\text { 4. Aspectos que influyen en la } \\
\text { implicación/dedicación/aprendizaje } \\
\text { vinculado al uso de la app }\end{array}$ & $\begin{array}{l}\text { 4.1. Motivación } \\
\text { 4.2. Formación/asesoramiento antes de usar la App } \\
\text { 4.3. Guía y desarrollo profesional durante uso de la App }\end{array}$ \\
\hline $\begin{array}{l}\text { 5. Sugerencias a introducir en } \\
\text { EVALOE-SSD }\end{array}$ & $\begin{array}{l}\text { 5.1. Revisión de las decisiones tomadas } \\
\text { 5.2. Revisión de la explicación escrita de los ítems (explicación/terminología) } \\
\text { 5.3. Recurrir de nuevo a las ayudas } \\
\text { 5.4. Imprimir las decisiones tomadas } \\
\text { 5.5. Aumentar la cantidad de vídeos } \\
\text { 5.6. Mejorar traducción inglés } \\
\text { 5.7. Cambios introducidos mientras se estaba usando/detección de mejoras técnicas } \\
\text { 5.8. Cambiar tamaño de la letra/iconos } \\
\text { 5.9. Incluir terminología igualdad de género } \\
\text { 5.10 Eliminar ítems puntuados sistemáticamente en verde } \\
\text { 5.11 Modificar el nivel de complejidad de los ítems }\end{array}$ \\
\hline $\begin{array}{l}\text { 6. Consecuencias del uso de la app y a } \\
\text { partir del uso en la incorporación de la } \\
\text { Metodología Conversacional }\end{array}$ & $\begin{array}{l}\text { 6.1. Uso del contenido de EVALOE-SSD (la Metodología Conversacional) en } \\
\text { diferentes áreas/asignaturas } \\
\text { 6.2. Ayuda a recordar prácticas olvidadas (lo que alguna vez hizo y dejó de hacer) } \\
\text { 6.3. Ayuda a reflexionar sobre las propias prácticas (qué hace el docente en el aula) } \\
\text { 6.4. Promueve cambios en la acción docente (qué ha empezado a hacer a partir del uso } \\
\text { de la App) } \\
\text { 6.5. Se evidencia un progreso de los alumnos } \\
\text { 6.6. Incorporar terminología/ reflexionar y comprender algún ítem en particular }\end{array}$ \\
\hline
\end{tabular}

curso de Educación Secundaria Obligatoria en los grupos de intervención y 104 en los grupos de comparación. Los grupos de los profesores que voluntariamente decidieron participar constituyeron los grupos de intervención, los demás grupos de los mismos centros y nivel constituyeron los grupos de comparación.

\section{Procedimiento}

La investigación, aprobada por los comités éticos de las dos universidades implicadas, se realizó durante el primer trimestre del curso 2020-21. Al inicio del curso se enviaron los consentimientos informados para que fueran firmados por las familias y los estudiantes de los grupos de intervención y comparación. A continuación, se desarrollaron las siguientes fases:

Fase 1. Pretest. Los estudiantes de todos los grupos participantes contestaron un Cuestionario de Autopercepción de Competencia Oral en una sesión de clase de tutoría.

Fase 2. Formación inicial intensiva. Al inicio del curso escolar, el profesorado del grupo de intervención participó en dos sesiones de formación online de dos horas de duración. En cada sesión los Investigadores Principales del 


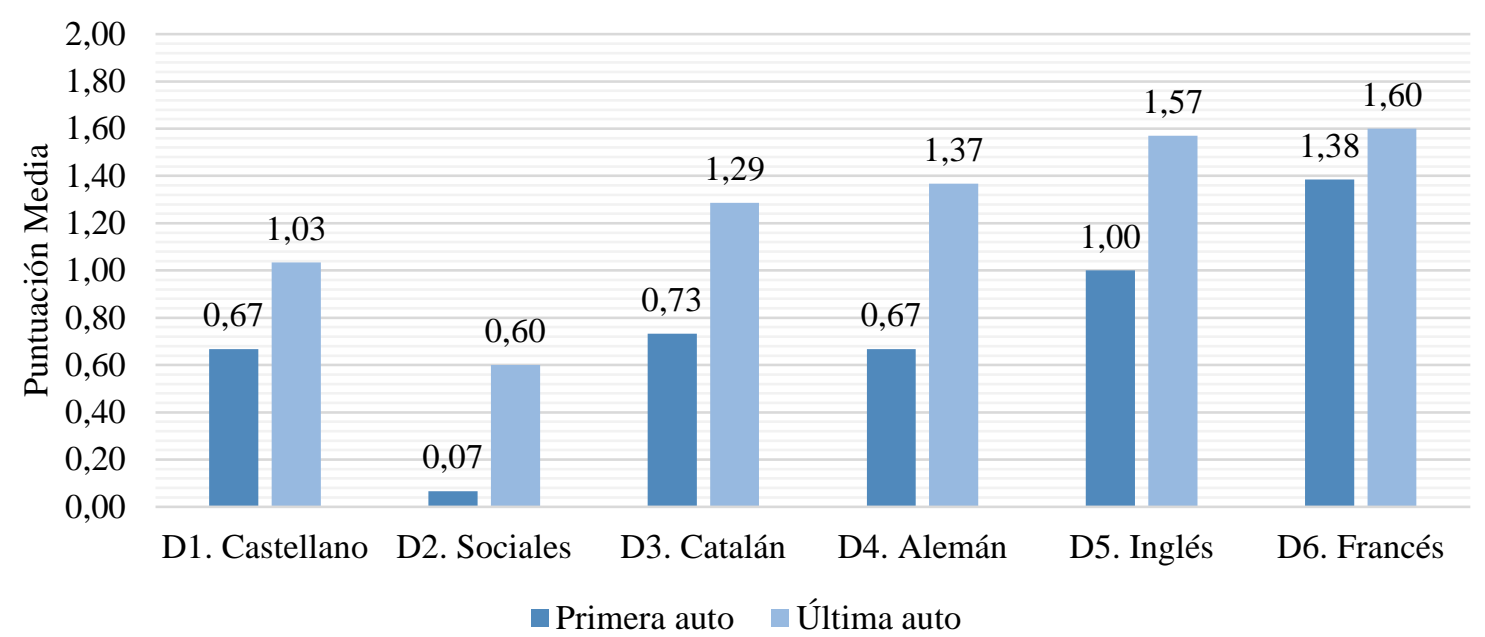

Figura 1. Valoración media de la primera y la última clase en las autoevaluaciones realizadas por los docentes

proyecto explicaron el uso y objetivos de la EVALOE-SSD.

Fase 3. Uso de la EVALOE-SSD. Durante 10 semanas, los 6 docentes utilizaron el recurso inteligente, describiendo sus clases y evaluando su comportamiento y el de su alumnado a partir de los recursos de la herramienta, generando nuevos recursos adaptados a su asignatura, condiciones y alumnado, tomando decisiones, reflexionando por escrito sobre la dinámica de las clases, la respuesta del alumnado e introduciendo cambios metodológicos en sus clases.

Fase 4. Postest. Los estudiantes de todos los grupos participantes contestaron un Cuestionario de Autopercepción de Competencia Oral.

Fase 5. Los profesores participantes rellenaron el Cuestionario de satisfacción al final del trimestre.

\section{Análisis de datos}

Se analizaron tanto los resultados sobre las autoevaluaciones realizadas por los profesores que incluye la EVALOE-SSD, los datos sobre autopercepción de las competencias comunicativas de los estudiantes antes (pretest) y después de la intervención (postest), como los datos de los cuestionarios de satisfacción final. Los paquetes estadísticos utilizados fueron el programa SPSS y el paquete Psych de R (RStudioTeam, 2020).

Los datos vinculados a las preguntas abiertas del cuestionario y las descripciones de docentes sobre sus clases han sido analizados cualitativamente en base a un sistema de categorías y códigos elaborado a partir de un proceso inductivo (ver Tabla 1).
Para realizar un análisis de contenido se utilizó la herramienta Atlas.ti 8 (Miles et al., 2014) y se generó el sistema de categorías mencionado. Dos jueces analizaron de manera independiente los datos y se calculó la fiabilidad inter-observador, siendo el coeficiente de Cohen's Kappa de .89 (Cerda \& Villarroel, 2008).

\section{Resultados}

Se presentan los resultados de la valoración de las clases por parte de los docentes mediante sus autoevaluaciones en la consecución de los distintos hitos que plantea el SIAP, los análisis del cambio en la autopercepción de las competencias orales de los estudiantes entre la evaluación inicial y la final, y las respuestas y reflexiones del profesorado acerca de las clases y de su experiencia de uso en la implementación del SIAP.

\section{Valoraciones de las clases por parte de los docentes a través de la EVALOE-SSD}

A partir del uso de la EVALOE-SSD, durante un periodo de 10 semanas, los docentes valoraron algunas de sus clases de diversas materias. La valoración se realizó en un rango de valores de 0 a 2 (los profesores señalan su progresión en una escala de tres colores rojo $=0$, naranja $=1$ y verde $=$ 2). La Figura 1 permite apreciar como la valoración de los docentes de sus acciones y de las de su alumnado, a su propio juicio, se incrementa positivamente de forma estadísticamente significativa a lo largo del proceso de formación pasando de una media en la sesión inicial de $\mathrm{M}=.75$ 


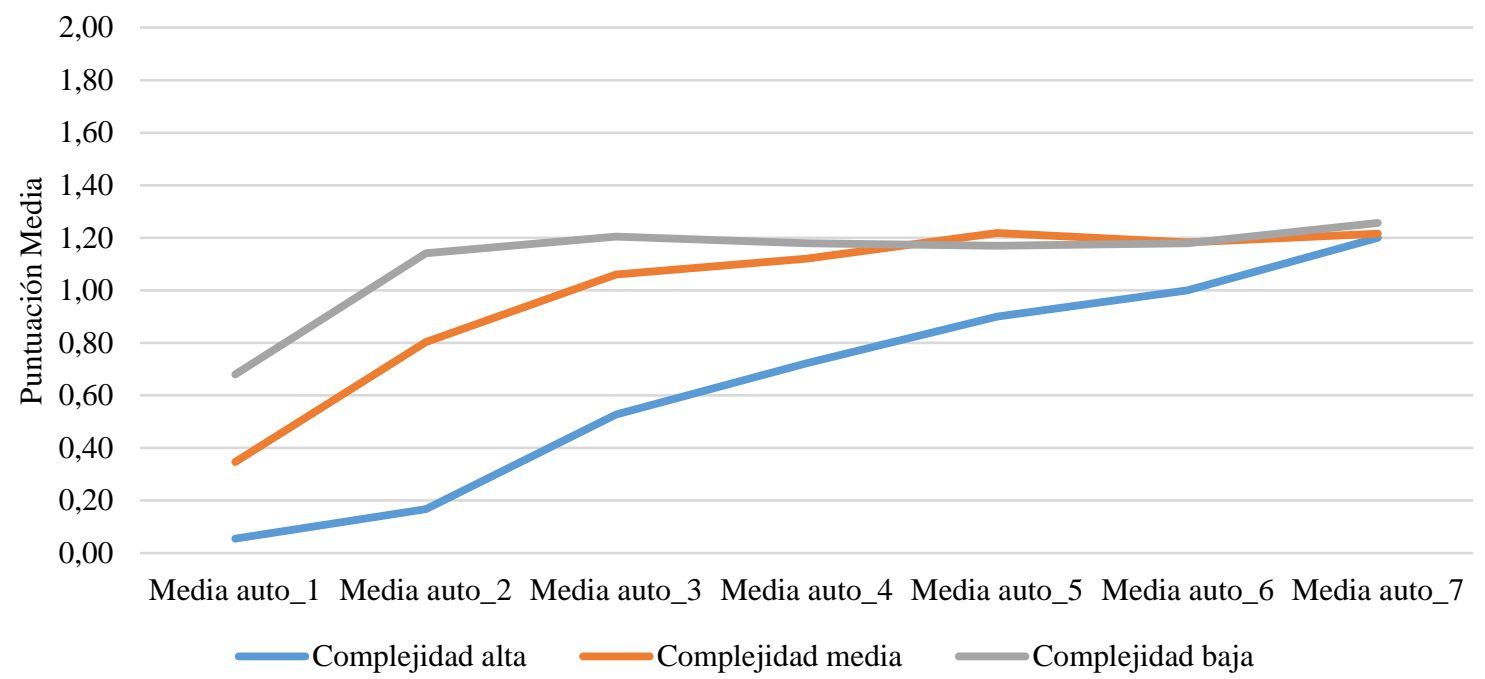

Figura 2. Valoración media de las clases por parte de los docentes en función del nivel de complejidad

$(\mathrm{DE}=.43)$ a una media en la sesión final de $\mathrm{M}=1.24(\mathrm{DE}=.38) t(5)=7.01, p=.01$. Debido a que cada asignatura era impartida generalmente por un solo profesor no fue posible estimar ni separar la variabilidad que depende de la materia y la que depende del profesor.

Asimismo, la evolución de las valoraciones agrupando los ítems por nivel de complejidad, muestra como, inicialmente, la valoración de los ítems más complejos es más baja que la de los menos complejos, diferencia que desaparece al final del periodo de formación (ver Figura 2).

Respaldando la autovaloración que hacen los docentes de su práctica, el análisis de la evolución de estos docentes (donde se compara la primera y última sesión), muestra una mejora en la implementación de las distintas estrategias de comunicación oral en todas las asignaturas, como puede verse en la Figura 3. A su vez se observa como el alumnado, a juicio de los docentes, fue mejorando en el uso de las estrategias entrenadas, hallándose una fuerte correlación entre las acciones de docente y alumnado tanto en la primera sesión $\mathrm{r}=.98, p<.01$ como en la última $\mathrm{r}=.92, p<.01$.

\section{Competencia oral autopercibida del alumnado}

Se solicitó a los estudiantes la autoevaluación de sus competencias comunicativas en las dimensiones de gestión de interacción, prosodia, cohesión textual y estrategias argumentativas. La evaluación se realizó al inicio (pretest) y al final de la intervención (postest), tanto en los grupos de comparación como en los grupos de intervención.
La fiabilidad del cuestionario, estimada mediante el coeficiente alfa, fue de .71 en el pretest y de .72 en el postest. Respecto a su estructura, se evaluó la bondad de ajuste a un modelo unidimensional mediante mínimos cuadrados ponderados diagonalizados, tanto en el pretest $\left(\chi^{2} \quad(104)=132.63, \quad p=.031, \quad \mathrm{CFI}=.95\right.$, TLI $=.94$, RMSEA=.04), como en el postest $\left(\chi^{2}\right.$ $(104)=111.83, \quad p=.282, \quad$ CFI $=.997, \quad$ TLI $=.99$, RMSEA=.02), lo que sugiere una bondad de ajuste aceptable (Hu \& Bentler, 1999 consideran que un modelo presenta una bondad de ajuste adecuado cuando RMSEA<.06, CFI y TLI $>.95$, siendo algo más restrictivos que Bentler, 1990 que considera la bondad de ajuste aceptable cuando los valores de RMSEA<.08 y los índices incrementales CFI y TLI>.90). Si bien, una estructura más compleja contemplando las cuatro dimensiones evaluadas permite mejorar la bondad de ajuste, en el pretest $\left(\chi^{2}(98)=107.64, p=.237\right.$, CFI $=.98$, TLI $=.98$, RMSEA $=.03)$ y en el postest $\left(\chi^{2} \quad(98)=94.133, p=.592, \quad\right.$ CFI $=1.0, \quad$ TLI $=1.0$, RMSEA=.01), razón por la cual se decidió estimar las puntuaciones factoriales de cada dimensión para posteriores análisis.

A partir de las puntuaciones factoriales se contrastó mediante un ANOVA de medidas repetidas si las diferencias entre las puntuaciones del pretest respecto al postest eran o no significativamente mayores en el grupo de intervención en las cuatro competencias evaluadas. La prueba de efectos intrasujetos reveló un efecto estadísticamente significativo del entrenamiento en el grupo de intervención $\mathrm{F}(1,235)=4.819, \mathrm{MC}=$ 

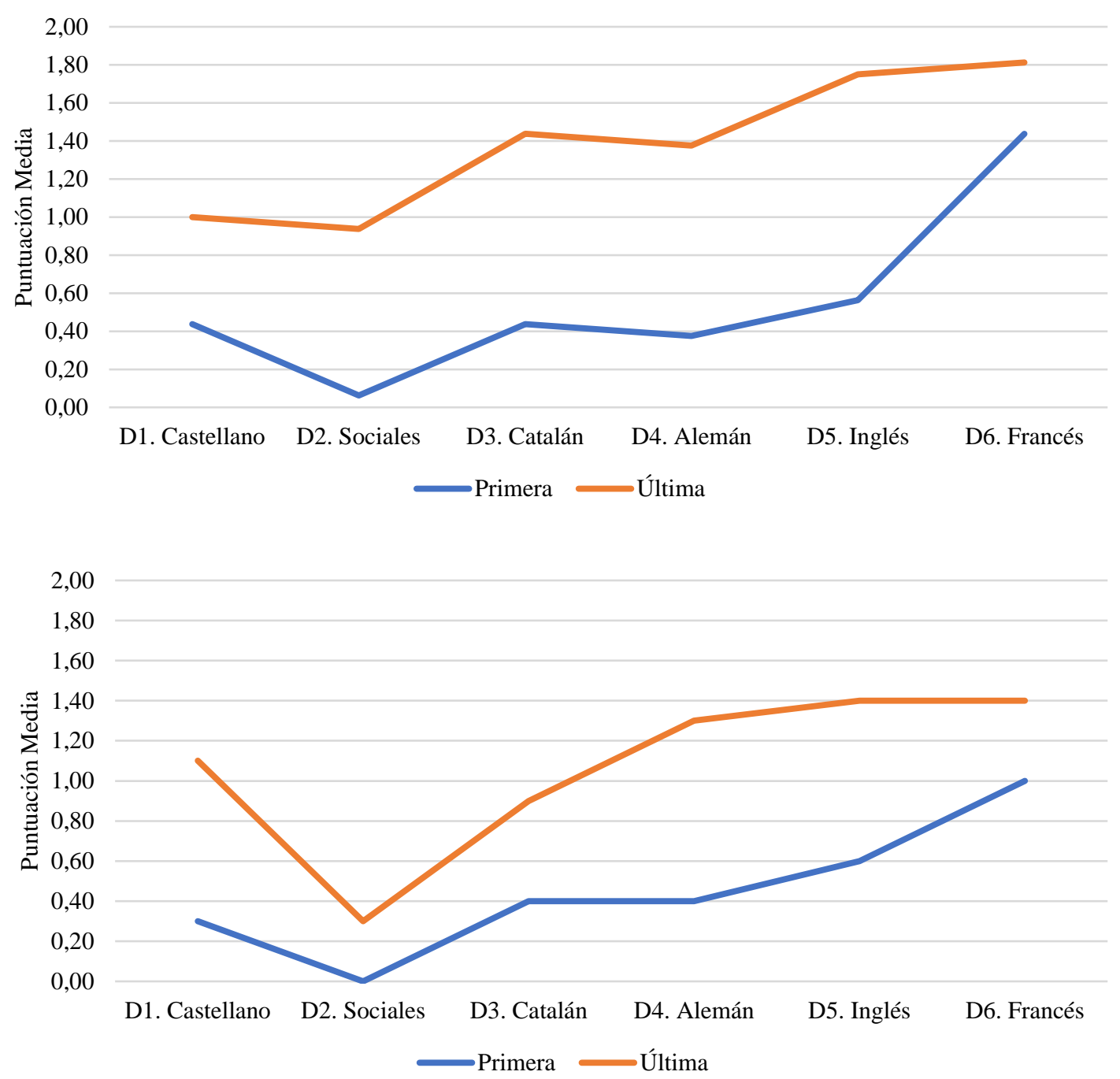

Figura 3. Evolución en las acciones de docentes entre la primera y última sesión (grafico superior) y evolución en las acciones de alumnado entre la primera y última sesión (gráfico inferior)

6.516, $p=.025, \eta_{\text {parcial }}^{2}=.027$ (en esta comparación la prueba de Levene no fue estadísticamente significativa $p=.288$, asumiéndose el supuesto de igualdad de varianza). La medida en el grupo de comparación revela un decremento en las puntuaciones factoriales $\mathrm{M}=-.35$ ( $\mathrm{DE}=2.43$ ), mientras que en el grupo de intervención las puntuaciones factoriales aumentaron $\mathrm{M}=.43$ $(\mathrm{DE}=2.19)$.

El ANOVA reveló, además, que la mejora con la intervención en el grupo de intervención se produjo por igual en las cuatro competencias evaluadas, ya que no se observaron diferencias estadísticamente significativas entre las cuatro competencias evaluadas $\mathrm{F}(3, \quad 522)=.056$, $\left.\mathrm{MC}=.019, p=.996, \eta_{\text {parcial }}^{2}<.001\right)$. Si bien en este contraste la prueba de esfericidad de Mauchly fue significativa $\left(\mathrm{W}=.888, \chi^{2}(5)=20.424, p=.001\right)$, se obtiene el mismo resultado al inspecionar las estimaciones corregidas de Greenhouse-Geisser ( $p=.994)$, Huynh-Feldt $(p=.995)$ o límite inferior $((p=.884)$.

Finalmente, como se puede apreciar en la Figura 4, la homogeneidad en la autopercepción de las competencias, se obtuvo, tanto en el grupo de intervención como en el de comparación, ya que la interacción entre competencias $\mathrm{x}$ grupo no fue estadísticamente significativa $\mathrm{F}(3$, 522) $=2.056, \quad \mathrm{MC}=1.800, \quad p=.105, \quad \eta_{\text {parcial }}^{2}=.012$; Greenhouse-Geisser $p=.110$, Huynh-Feldt $p=.109$ y límite inferior $p=.153$.

\section{Reflexiones de los docentes en las descripciones de las clases}

La primera actividad que realizan los docentes cuando utilizan la EVALOE-SSD es una descripción de la sesión de clase sobre la que se disponen a reflexionar. A continuación, se presentan resultados 

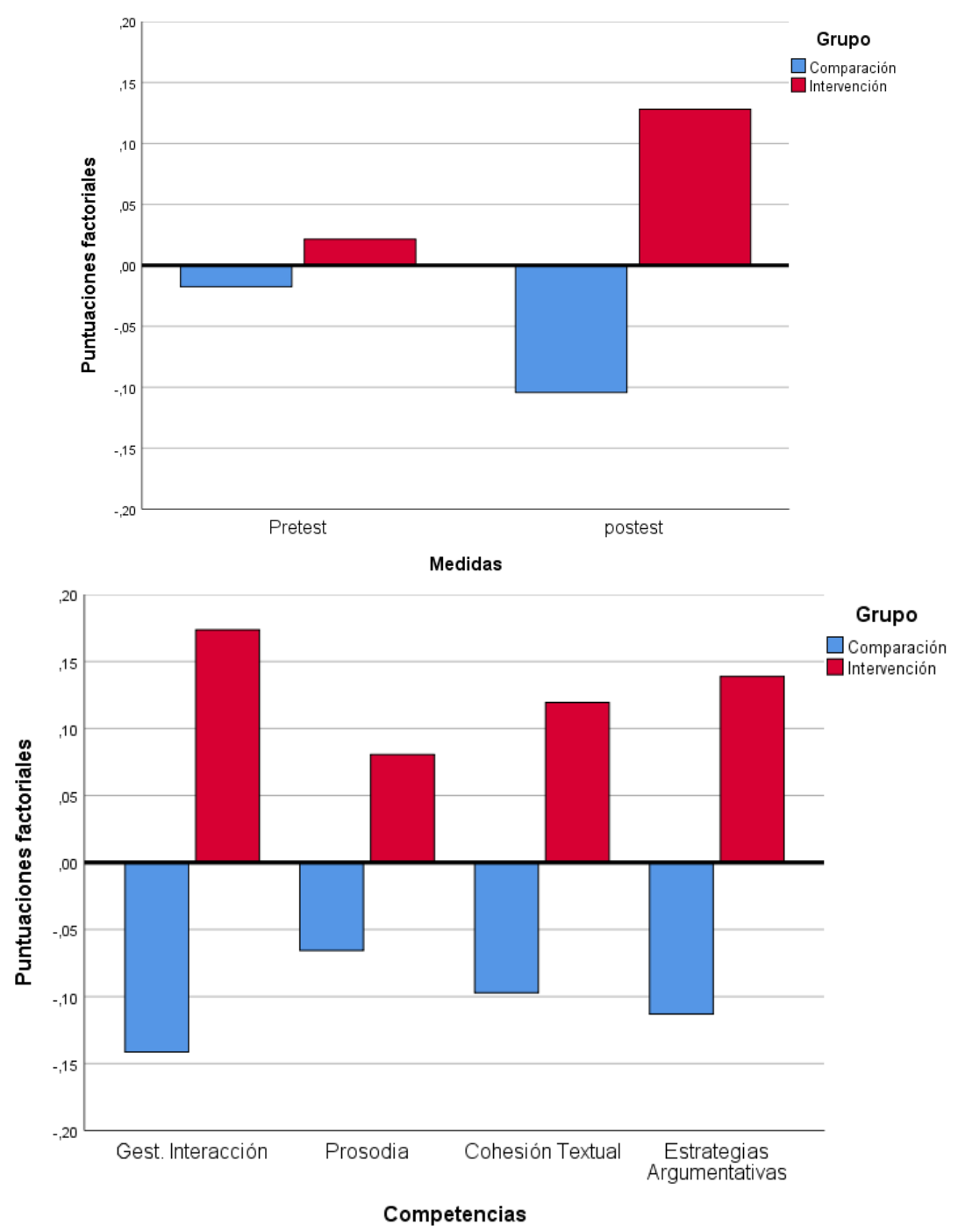

Figura 4. Puntuaciones factoriales en los grupos de comparación e intervención en el pretest y postest (gráfico superior) y puntuaciones por dimensión en postest (gráfico inferior)

relacionados con estas descripciones que hicieron los docentes de los grupos de intervención al inicio de cada una de las autoevaluaciones con la EVALOE-SSD. Los profesores que participaron en la condición de comparación no realizaron estas descripciones debido a que no utilizaron la herramienta digital.

El análisis cualitativo de todas las descripciones realizadas por los seis docentes en base al sistema de categorías presentado en la Tabla 1 indica que, de las 59 citas identificadas, 43 están relacionadas con la capacidad del SIAP para ayudar a reflexionar sobre las propias prácticas (Código 6.3. Ayuda a reflexionar sobre las propias prácticas -qué hace el docente en el aula-) (por ejemplo, «previamente recordamos cómo podemos expresar nuestras opiniones y mostrar cómo estamos de acuerdo o en desacuerdo con lo que dicen las otras personas», «comienzan aportando argumentos sólidos para defender sus posiciones con entusiasmo»), 10 citas giran alrededor de los cambios que ha promovido la herramienta inteligente en sus prácticas en el aula (Código 6.4. Promueve cambios en la acción docente (qué ha empezado a hacer a partir del uso de la App) (por ejemplo, «antes de empezar la sesión se hace un breve recordatorio sobre lo que había que hacer para entenderse y participar en la conversación, estoy mucho más pendiente de cosas y detalles que antes me pasaban desapercibidos, cambié la disposición de las mesas y se pusieron en forma de "U", de momento ellos no saben que he introducido algunos cambios al inicio y al final de la clase para conseguir que todos participen»), 5 con la reflexión sobre algunos ítems en particular (Código 6.6. Incorporar terminología / reflexionar y comprender algún ítem en particular) (por 
ejemplo, «previamente reflexionamos sobre cómo podemos notar cuándo podemos intervenir en una conversación sin tener que levantar la mano para pedir turno y vamos probando ponerlo en práctica, les he ayudado a tomar conciencia de que es importante mirarse cuando se hablan o se preguntan algo»), y 1 con el progreso del alumnado (Código 1.2. Referencia a la utilidad de las ayudas) (por ejemplo, «hemos profundizado y reformulado algunos aspectos surgidos de la conversación que no estaban preparados previamente»).

\section{Valoración de la experiencia de uso del asistente pedagógico inteligente}

A continuación presentamos los resultados obtenidos a partir del análisis de las respuestas de los docentes al cuestionario de satisfacción contestado al final del proceso de formación del profesorado, que contenía preguntas abiertas y cerradas.

En cuanto a las preguntas sobre la utilidad del tutorial, los seis docentes contestaron afirmativamente. Respecto a la redacción de los ítems todos contestaron que no introducirían ningún cambio. También coincidieron todos en que no cambiarían nada de la descripción de cada uno de los ítems. En relación con la pregunta de si les parecía que sobraban ítems, la mitad contestó afirmativamente y la otra contestó que no. Todos los docentes consideraron que las ayudas en formato escrito (preguntas de reflexión) eran útiles y 5 afirmaron que las ayudas en formato vídeo también lo eran.

Respecto a las preguntas abiertas, nos centraremos en las tres en las que se pedía a los docentes que indicasen: 1) por qué consideraban adecuado, si era el caso, que la primera vez que se valoraba un ítem no se le pudiese asignar el color verde; 2) los tres aspectos que mejor valoraban de la herramienta, 3) comentarios que considerasen que podían ser útiles para mejorar la herramienta.

En relación con la primera, algunas de las respuestas fueron: «te hace ver, y eso es muy bueno, que aunque seas profesora de lenguas y pienses que la parte oral la tienes cubierta, es un engaño, y ves que puedes mejorar muchísimo este campo»; «es un aprendizaje; porque ayuda a reflexionar, evidentemente; porque sirve como recordatorio de que se trata de mejorar la calidad de la comunicación y expresión oral, es decir, que partimos de un punto mejorable»; «creo que algunos ítems son la esencia del trabajo del docente y que los docentes los incorporan a su práctica de forma natural e intuitiva, eso sí, otros lo son menos y está bien que vengan así señalados».

Respecto a la segunda pregunta, relacionada con los tres aspectos que mejor valoraban de la herramienta, algunas de las respuestas fueron: «es fácil de utilizar, intuitiva»; es bastante sintética y completa a la vez»; «me gusta que me obligue a reflexionar sobre mi práctica docente, que me paute las dimensiones con los diferentes ítems y que hagamos un encuentro con todos los docentes participantes para poner en común las actividades que vamos desarrollando y los logros conseguidos»; «saber qué hago y qué me falta (reflexión), darme cuenta de la importancia de algunos ítems que tenía descuidados (participación de todo el alumnado), poder llegar a dar clases mucho más enriquecedoras a nivel oral»; «invita a reflexionar sobre el planteamiento y la manera de hacer las clases; la reflexión que conlleva, la exigencia de estar pendiente del uso de la lengua oral, la mejora como resultado de las dos anteriores; la explicación de lo que incluye cada ítem»; «el hecho de que tengas que concentrarse en tres aspectos cada vez; la autoevaluación y, por tanto, reflexión de aquella actividad realizada»; «creo que el uso de la metodología EVALOE puede mejorar sus clases y la gestión del aula; «cualquier aclaración, material de ayuda, actividades, vídeos es un recurso muy bienvenido».

Finalmente, las respuestas a la tercera pregunta, vinculada a comentarios que considerasen que podían ser útiles para mejorar la herramienta, los docentes escribieron: «creo que es una herramienta que puede ser muy útil para utilizar al menos una temporada, pero quizás con una evaluación menos frecuente»; «quizás se podría matizar los ítems relativos a la participación espontánea, la gestión autónoma de la palabra y a la cuestión del debate».

\section{Discusión}

En este estudio se muestran los resultados del diseño e implementación de un Sistema Inteligente destinado a la Asistencia Pedagógica (SIAP) al profesorado. La aplicación práctica de la EVALOE-SSD se realizó durante 10 semanas y 
tenía como objetivo facilitar la labor docente para promover el desarrollo de la competencia oral de estudiantes de primer y tercer curso de Educación Secundaria Obligatoria en diferentes centros de dos comunidades autónomas españolas.

Respecto a la primera pregunta de investigación sobre la utilidad de la herramienta para mejorar la práctica docente, nuestros resultados muestran que los docentes han aprendido a través de la EVALOE-SSD, ya que las valoraciones que hacen de sus acciones van mejorando progresivamente a lo largo del trimestre. A su vez, el SIAP es sensible a las diferencias entre docentes, en concreto, en la percepción que cada uno tiene de sus clases. Algunos docentes hacen valoraciones relativamente altas desde el inicio, como es el caso de las profesoras de Inglés y Francés, y otros hacen una autoevaluación inicial mucho más baja, como es el caso del profesor de Sociales. Un aspecto a resaltar es que, en general, la evaluación de los ítems más complejos es más baja inicialmente que la de los menos complejos. Este resultado indica que las respuestas de los docentes validan la agrupación de los ítems de la EVALOE-SSD en cuanto a su nivel de complejidad. ¿Qué significa que son ítems más complejos? ¿Qué reto plantean al docente? Tal como se ha defendido en trabajos previos (Gràcia et al., 2020a, 2020b), la complejidad está directamente relacionada con la naturaleza y la cantidad de los elementos o factores a tener en cuenta simultáneamente en la gestión, organización y estrategias utilizadas durante las clases para promover una participación activa del alumnado (Mercer et al., 2019).

El cuestionario incluye seis ítems de alta complejidad, uno en cada una de las dimensiones, excepto en la dimensión de diseño instruccional, en el que hay dos (el alumnado autoevalúa su competencia comunicativa, y evalúo la competencia comunicativa del alumnado). En los dos casos se trata de acciones vinculadas a la evaluación. Conseguir que el alumnado aprenda a autoevaluar su competencia significa que el profesorado utiliza estrategias diversas para compartir con el alumnado los objetivos de lengua oral, así como revisar conocimientos previos acerca de contenido lingüístico, que son dos de los ítems de complejidad media. ¿A qué nos referimos con contenido lingüístico? Estamos hablando de acciones tales como referirse a las normas de comunicación o a gestionar los turnos con la finalidad de que el alumnado participe en las conversaciones y las discusiones. Otro de los ítems de máxima complejidad es el referido a la adopción de un formato de interacción en red durante las actividades de conversación y discusión. En este caso la complejidad viene dada porque supone que está implicado todo el alumnado y el docente, y que, durante estas interacciones en red, el alumnado tiene que demostrar que puede al mismo tiempo mostrar habilidades como aportar información (describir, argumentar..), obtener información (formular preguntas reales, retóricas, a sus compañeros, al docente, a todos), sintetizar lo dicho hasta el momento, asegurarse de que le escuchan, de que no alarga demasiado su turno, estar pendiente de los otros compañeros que también quieren participar, incluso de algunas expresiones no verbales que le ayudan a ir modelando o modificando su discurso.

En definitiva, demostrar competencia para regular su conducta durante una actividad dinámica, improvisada, viva, en la que la oportunidad de aprender mientras se participa en una discusión entre los compañeros y del docente son infinitas (Mercer et al., 2019, 2020; Romero et al., 2014).

¿Qué es lo que ha ayudado al profesorado a cambiar, a mejorar, a aprender? No se trata únicamente de la posibilidad de revisar información, sino de transformar esta información en conocimiento (Son, 2018; Thorn, 2002). La EVALOE-SSD permite a los docentes leer las descripciones de cada dimensión e ítem, así como las preguntas de reflexión que aparecen como uno de los recursos para evaluar de manera más informada sus clases. También pueden visionar los vídeos de otros profesores interactuando con alumnos y alumnas, como muestra de buenas prácticas.

Los resultados indican que todos estos recursos han posibilitado la reflexión sobre su propia práctica. Esto se detecta tanto en el análisis de las descripciones de las sesiones como en las respuestas al cuestionario de satisfacción final. Las reflexiones que escriben los docentes en este cuestionario significan que realmente han 
revisado y ampliado sus concepciones sobre lo que es enseñar y aprender lengua oral, han modificado sus esquemas de conocimiento en sentido positivo, que ahora son más complejos, lo que es otra manera de decir que han aprendido significativamente (Ausubel, 2000; Coll, 2010; Pozo, 2020). Otros docentes afirman directamente que «es un aprendizaje». No es frecuente que docentes de Educación Secundaria realicen este tipo de afirmaciones de reflexión sobre su práctica y su aprendizaje (James \& McCormick, 2009), como tampoco lo es que la participación en una formación suscite propuestas de mejora, tanto respecto a la herramienta que están usando como en relación con la manera de usarla, aunque sí hay estudios previos que lo indican (Cartwright, 2011; Sedova, 2017; Schön, 1987). Los resultados parecen mostrar que la SIAP pone el acento en ayudar al docente en su tarea de guía al proceso de aprendizaje del alumnado.

Los resultados obtenidos a partir del análisis de las descripciones iniciales son un reflejo de la capacidad de la SIAP para generar reflexiones sobre la enseñanza y el aprendizaje de la lengua oral en el marco de las actividades que se realizan en el aula en asignaturas muy diversas, poniendo el foco en la conversación y en la discusión conjunta como elemento clave para la construcción de conocimiento.

En conjunto, el análisis de las descripciones iniciales y de las respuestas al cuestionario final indican que, durante el proceso de desarrollo docente y al final de este, los docentes cambian progresivamente la manera de referirse a sus clases, de describirlas, de usar los conceptos teóricos que están incluidos en la herramienta, que inicialmente no usaban en las descripciones, y cambian la manera de describir lo que hacen los alumnos en sus clases. Esto es aprendizaje (James \& McCormick, 2009).

En la línea de estudios recientes que abordan la enseñanza y el aprendizaje de la lengua oral en L1 en Educación Secundaria (para una revisión ver Wurth et al., 2019), la segunda pregunta de investigación que ha dirigido este trabajo está relacionada con la percepción que tiene el profesorado sobre la mejora de la competencia oral de su alumnado a través del uso de la EVALOE-SSD. A juicio del profesorado, su uso ha contribuido a la mejora de la competencia oral del alumnado, tal y como se recoge en las valoraciones. Se entiende que ha sido posible gracias al progreso de los docentes. Así, nuestros resultados permiten diferenciar entre el efecto de la herramienta en el profesorado y en el alumnado, lo cual confirma que ha facilitado al docente, durante su proceso formativo, no sólo la evaluación de su práctica y sus estrategias, sino también de los progresos del alumnado. Estos resultados coinciden con los hallados por otros autores, aunque utilizando instrumentos menos complejos (Wong et al., 2017).

Como ya se ha indicado en apartados anteriores, una parte de los ítems de la EVALOESSD se refieren a conductas comunicativas de los alumnos, por lo que la mejora de las puntuaciones es otra manera de evaluar su progreso, como pueden ser los exámenes o los trabajos escritos, en este caso referida a la competencia en lengua oral. En cambio, la valoración de los ítems que hacen referencia a la propia conducta comunicativa, la de los profesores, a su juicio, supone una autoevaluación, un autoinforme, pero no por ello deja de tener valor. La EVALOE-SSD se encuentra entre el tipo de instrumentos cada vez más utilizados en la investigación educativa, que suponen un proceso de reflexión sobre la práctica que es esencial en el desarrollo docente (Fainholc et al., 2013).

Finalmente, respecto a las preguntas de investigación planteadas en relación con los cambios autopercibidos en competencia oral por los/as alumnos/as a lo largo del periodo de formación y sobre en qué dimensiones se detectan los avances más importantes, los resultados obtenidos indican, en conjunto, que la participación de los docentes en la formación ha contribuido a la reflexión metalingüística de los alumnos y alumnas de los grupos de intervención, en la línea de los resultados de estudios recientes que analizan cómo el alumnado universitario autoevalúa su competencia en comunicación oral (Campos et al., 2021).

En cuanto a los cambios en el tiempo (prepost intervención) en la percepción del alumnado de los grupos de comparación y de intervención relacionados con las dimensiones medidas (Gestión de la interacción, Prosodia, Cohesión textual y Estrategias argumentativas), nos interesa especialmente destacar que no se trata 
tanto de mostrar que la percepción del alumnado de los grupos de intervención coincide con la valoración realizada por los docentes, sino de mostrar cómo el uso del SIAP por parte de sus profesores, y por consiguiente de los cambios introducidos en las clases, ha provocado que el alumnado reflexione sobre sus competencias en lengua oral y cambie su percepción inicial. El SIAP plantea la participación en clase como una oportunidad para contribuir a la capacidad de autorregulación por parte del alumnado en las conversaciones (Clarke et al., 2016; Mercer et al., 2019; Nami et al., 2016). En este sentido, la herramienta permite que los docentes cambien su manera de entender el aprendizaje, la enseñanza y su rol, y esto, a su vez, tiene un efecto en la autopercepción del alumnado, en las dinámicas de interacción que se generan y en cómo aprender a autorregular sus emociones (Jiménez-Blanco et al., 2020).

Tras 10 semanas, la intervención ha impactado en la percepción del alumnado de algunas habilidades relacionadas con la gestión de la conversación, como la toma de conciencia de la importancia de hablar en clase (Boyd \& Markarian, 2015), o de que hay otras maneras más sutiles o indirectas de hacerlo (tono de voz del interlocutor que está hablando, gesticulación, expresión verbal, e incluso contenido de sus intervenciones). También se observa una mejora en estrategias argumentativas, concretamente en la autopercepción en los grupos de intervención, se trata de una habilidad que se vincula claramente a dos de los ítems de alta complejidad del cuestionario, uno referido al profesorado y otro al alumnado.

La EVALOE-SSD fue ideada para la mejora de la competencia oral, por lo que su uso está limitado a esta competencia comunicativa. En futuros desarrollos podrían incluirse otros módulos en los que se incluya la competencia escrita y su evaluación progresiva (CáceresSerrano \& Alvarado-Izquierdo, 2018). Para ello deberán elaborarse los estándares de aprendizaje imprescindibles para una docencia eficaz en el desarrollo de estas nuevas competencias.

En cuanto a los resultados que se presentan, deben tomarse como provisionales, es necesario un mayor esfuerzo investigador para determinar los factores que afectan a la eficacia de la herramienta, si es igualmente efectiva en grupos con problemas de aprendizaje, y es de gran interés en el desarrollo de la herramienta evaluar su utilidad para el desarrollo del lenguaje signado, cuestión que será abordada en la siguiente versión del instrumento, aunque ya ha sido utilizado en contextos de uso de lengua de signos en Educación Infantil (Gràcia \& Lacerda, 2019) con resultados alentadores. Puesto que la eficacia de toda herramienta pedagógica se relaciona estrechamente con el grado de compromiso del profesorado, es una cuestión también pendiente el evaluar cómo impacta este compromiso en el éxito de la herramienta. De la misma manera que sería interesante contrastar las autoevaluaciones que hacen los docentes de sus clases con las evaluaciones que hace un observador externo, como ya se ha hecho en trabajos anteriores con maestras de Educación Infantil y Primaria (Gràcia et al., 2020a, 2020b), con resultados positivos.

El estudio realizado sobre una experiencia de formación de un grupo de profesorado de Educación Secundaria en el uso de un Sistema Inteligente de Asistencia Pedagógica ha mostrado resultados prometedores, tanto para monitorizar el proceso de aprendizaje y toma de decisiones del profesorado ante el reto de la enseñanza explícita de las competencias de comunicación oral en el aula, como para la mejora de estas competencias en el propio alumnado.

Al tratarse de una herramienta abierta y flexible que permite generar recursos educativos orientados a objetivos pudiéndose aplicar en todas las asignaturas, las posibilidades de implementación en el aula son ilimitadas.

\section{Referencias}

Arnott, D., \& Pervan, G. (2016). A critical analysis of decision support systems research. In L. P. Willcokcs, C. L. P. Sauer, \& M. C. Lacity (Eds.), Formulating research methods for information systems (pp. 127-168). Palgrave Macmillan. https://doi.org/10.1057/9781137509857

Ausubel, D. (2000). The adquisition and retention of knowledge. Kluwer Academic Publishers. https://doi.org/10.1007/978-94-015-9454-7 
Bentler, P. M. (1990). Comparative fit indexes in structural models. Psychological Bulletin, 107(2), 238. https://doi.org/10.1037/0033-2909.107.2.238

Berson, Y., Halevy, N. Shamir, B., \& Erez, M. (2015). Leading from different psychological distances: A construal-level perspective on vision communication, goal setting, and follower motivation. The Leadership Quarterly, 26(2),143-155. https://doi.org/10.1016/j.leaqua.2014.07.011

Boyd, M. P., \& Markarian, W. C. (2015). Dialogic teaching and dialogic stance: Moving beyond interactional form. Research in Teaching of English, 49(3), 272-296. https://doi.org/10.1080/09500782.2011.597861

Cáceres Serrano, P., \& Alvarado Izquierdo, J. M. (2019). Evaluación de la comprensión lectora mediante un modelo de diagnóstico cognitivo. Revista Iberoamericana de Diagnóstico y Evaluación - e Avaliação Psicológica, 2(51), 149-162. https://doi.org/10.21865/RIDEP51.2.11

Campos, I., Colón, M. J., Sampériz (2021). Diseño de un cuestionario para valorar la competencia en comunicación oral del alumnado de Magisterio. Educação \& Formação, 6 (1), e3490-e3490. https://doi.org/10.25053/redufor.v6i1.3490

Capa-Aydin, Y., Sungur, S., \& Uzuntiryaki, E. (2009). Teacher self-regulation: Examining a multidimensional construct. Educational Psychology, 29(3), 345-356. https://doi.org/10.1080/01443410902927825

Cartwright, L. (2011). How consciously reflective are you? In D. McGregor, \& L. Cartwright (Eds.), Developing reflective practice: $A$ guide for beginning teachers (pp. 55-68). McGraw-Hill Education. https://bit.ly/34dTWeE

Cerda, J., \& Villarroel, L. (2008). Evaluación de la concordancia inter-observador en investigación pediátrica: Coeficiente de Kappa. Revista Chilena De Pediatría, 79(1), 54-58.

https://doi.org/10.4067/S037041062008000100008

Chatzistamatiou, M., Dermitzaki, I., \& Bagiatis, V. (2014). Self-regulatory teaching in mathematics: Relations to teachers' motivation, affect and professional commitment. European Journal of Psychology of Education, 29(2), 295-310. https://doi.org/10.1007/s10212-013-0199-9

Clarke, S. N., Resnick, L. B., \& Penstein-Rosé, C. (2016). Dialogic instruction: A new frontier. In L. Corno, \& E. M. Anderman (Eds.), Handbook of Educational Psychology (pp. 378-389). Routledge. https://doi.org/10.4324/9781315688244

Coll, C. (2010). Enseñar y aprender, construir y compartir: Procesos de aprendizaje y ayuda educativa. In C. Coll (Ed.), Desarrollo, aprendizaje y enseñanza en la educación secundaria (pp. 31-61). Graó. https://bit.ly/3yvzy6N

Compton, L. K. (2009). Preparing language teachers to teach language online: A look at skills, roles, and responsibilities. Computer Assisted Language Learning, 22(1), 73-99. https://doi.org/10.1080/095882208026138

Fainholc, B., Nervi, H., Romero, R., \& Halal, C. (2013). Teacher training and pedagogical use of ICT [La formación del profesorado y el uso pedagógico de las TIC]. RED Revista de Educación a Distancia, 38, 1-14. https://bit.ly/2T8tfpz

Gewerc, A., \& Montero, L. (2015). Professional knowledge and digital competence in teacher education. The case of the Master Degree in Primary Education [Conocimiento profesional y competencia digital en la formación del profesorado. El caso del Grado de Maestro en Educación Primaria]. Revista Latinoamericana de Tecnología Educativa, 14(1), 31-43. https://bit.ly/3wpVEG4

Girvan, C., Conneely, C., \& Tangney, B. (2016). Extending experiential learning in teacher professional development. Teaching and Teacher Education, 58, 129-139. https://doi.org/10.1016/j.tate.2016.04.009

Gràcia, M., Galván-Bovaira, M. J., Sánchez-Cano, M., Vega, F., Vilaseca, R., \& Rivero, M. (2015a). Valoración de la enseñanza de la lengua oral. Escala EVALOE. Graó.

Gràcia, M., Vega, F., \& Galván-Bovaira, M. J. (2015b). Developing and testing EVALOE: A tool for assessing spoken language teaching and learning in the classroom. Child 
Language Teaching Therapy, 31(3), 287-304. https://doi.org/10.1177/0265659015593608

Gràcia, M., \& Lacerda, C. (2019). La formación de maestros y la promoción de la competencia comunicativa de alumnos sordos. Revista Educação Especial, 33. http://dx.doi.org/10.5902/1984686X48495

Gràcia, M., Casanovas, J., Riba, C., Sancho, M.R., Jarque, J., Casanovas, J. \& Vega, F. (2020a). Developing a digital application (EVALOEDSS) for the professional development of teachers aiming to improve their students' linguistic competence. Computer Assisted Language Learning, 1-26. https://doi.org/10.1080/09588221.2019.17076 90

Gràcia, M., Jarque, M.J., Riba, C., \& Vega, F. (2020b). Uso de una herramienta digital como recurso de desarrollo profesional para mejorar la competencia comunicativa oral de alumnos de educación infantil y primaria. Revista de investigación en Logopedia, 10(2), 135-149. https://doi.org/10.5209/rlog.67125

Gràcia, M., Jarque, J., Astals, M., \& Rouaz, K. (2020c). Desarrollo y evaluación de la competencia comunicativa en la formación inicial de maestros. Revista Iberoamericana de Educación Superior, 11(30), 115-136. https://doi.org/10.22201/iisue.20072872e.202 0.30 .591

Gràcia, M., Vega, F., Bitencourt, D., Vinyoles, N., \& Jarque, M.J. (2021a). Retos en la formación inicial de profesorado de infantil y primaria: La competencia oral. Revista Mexicana de Investigación Educativa, 26(88), 195-224. http://hdl.handle.net/2445/173311

Gràcia, M., Vega, F., Jarque, S., Adam, A. L., \& Jarque, J. (2021b). Teaching practices for developing oral language skills in Catalan schools. Cogent Education, 1-20. https://doi.org/10.1080/2331186X.2021.1935647

Hu, L. T., \& Bentler, P. M. (1999). Cutoff criteria for fit indexes in covariance structure analysis: Conventional criteria versus new alternatives. Structural Equation Modeling: A Multidisciplinary Journal, 6(1), 1-55. https://doi.org/10.1080/10705519909540118

Hughes, J. E., Liu, S., \& Lim, M. (2016). Technological modeling: Faculty use of technologies in preservice teacher education from 2004 to 2012. Contemporary Issues in Technology and Teacher Education, 16(2), 184-207. https://bit.ly/3fcS5gJ

Instefjord, E. J., \& Munthe, E. (2017). Educating digitally competent teachers: A study of integration of professional digital competence in teacher education. Teaching and Teacher Education, 67, 37-45.

https://doi.org/10.1016/j.tate.2017.05.016

James, M., \& McCormick, R. (2009). Teachers learning how to learn. Teaching and Teacher Education, 25(7), 973-982.

https://doi.org/10.1016/j.tate.2009.02.023

Jiménez Blanco, A., Sastre Llorente, S., Artola González, T., \& Alvarado Izquierdo, J. M. (2020). Inteligencia emocional y rendimiento académico: Un modelo evolutivo. Revista Iberoamericana de Diagnóstico y Evaluación - e Avaliação Psicológica, 3(56), 129-141. https://doi.org/10.21865/RIDEP56.3.10

Koh, L. H. (2019). TPACK design scaffolds for supporting teacher pedagogical change. Educational Technology Research and Development, 67(3), 577-595. https://doi.org/10.1007/s11423-018-9627-5

Könings, K. D., \& Gijselaers, W. H. (2015). Bringing learning to the workplace: A smartphone app for reflection and increased authenticity of learning. In A. Dailey-Hebert, \& K. S. Dennis, (Eds.), Transformative perspectives and processes in higher education (pp. 117-135). Springer. https://doi.org/10.1007/978-3-319-09247-8_7

Marope, M., Griffin, P., \& Gallagher, C. (2017). Future competences and the future of curriculum. A global reference for curricula transformation UNESCO-IBE. https://bit.ly/2XzeZXf

Mercer, N., Hennessy, S., \& Warwick, P. (2019). Dialogue, thinking together and digital technology in the classroom: Some educational implications of a continuing line of inquiry. International Journal of Educational Research, 97, 187-199. https://doi.org/10.1016/j.ijer.2017.08.007

Mercer, N., Marion, J., \& Warwick, P. (2020). Oracy education. En N. Mercer, R. Wegerif and L. Major (eds.). The Routledge International handbook of research on Dialogic Education. Routledge. 
https://doi.org/10.4324/9780429441677

Miles, M. B., Huberman, M., \& Saldaña, J. (2014). Qualitative Data Analysis: A methods sourcebook (3a ed.). Sage Publications.

Mosley-Wetzel, M., Maloch, B., \& Homan, J. V. (2017). Retrospective video analysis: A reflective tool for teachers and teacher educators. The Reading Teacher, 70(5), 533542. https://doi.org/10.1002/trtr.1550

Millard, W., \& Menzies, L. (2016) The State of Speaking in our Schools. Voice 21/LKMco.

Molinari, L., \& Mameli, C. (2013). Process quality of classroom discourse: Pupil participation and learning opportunities. International Journal of Educational Research, 62, 249-258.

https://doi.org/10.1016/j.ijer.2013.05.003

Nami, F., Marandi, S. S., \& Sotoudehnama, E. (2016). CALL teacher professional growth through lesson study practice: An investigation into EFL teachers' perceptions. Computer Assisted Language Learning, 29(4), 658-682. https://doi.org/10.1080/09588221.2015.1016439

Paredes, J., Guitert, M., \& Avi, B. (2015). Innovation and educational technology as the basis of initial teacher education for the renewal of teaching. RELATEC: Revista Latinoamericana de Tecnología Educativa, 14(1), 101-114.https://bit.ly/3wsofdL

Persico, D., Milligan, C., \& Littlejohn, A. (2015). The interplay between self-regulated professional learning and teachers' workpractice. Procedia-Social and Behavioral Sciences, 191, 2481-2486.

https://doi.org/doi:10.1016/j.sbspro.2015.04.590

Pozo, J. L. (2020). La educación está desnuda. Lo que deberíamos aprender de la escuela confinada. SM. https://bit.ly/3fEm0gJ

Prieto, L. P., Magnuson, P., Dillenbourg, P., \& Saar, M. (2017). Reflection for action: Designing tools to support teacher reflection on everyday evidence. https://bit.ly/34a5U9u

Reid, D. A., Simmt, E., Savard, A., Suurtamm, C., Manuel, D., Lin, T. W. J., ... Knipping, C. (2015). Observing observers: Using video to prompt and record reflections on teachers' pedagogies in four regions of Canada. Research in Comparative and International Education, 10(3), 367-382. https://doi.org/10.1177/1745499915580425

Reznitskaya, A. (2012). Dialogic teaching: Rethinking language use during literature discussions. The Reading Teacher, 65(7), 446456. https://doi.org/10.1002/ TRTR.01066

Romano, M., \& Schwartz, J. (2005). Exploring technology as a tool for eliciting and encouraging beginning teacher reflection. Contemporary Issues in Technology and Teacher Education, 5(2), 149-168. https://bit.ly/2SnEdaf

Romero, M., Guardia, L., Guitert, M., \& Sangra, A. (2014). Teachers' professional development through learning ecologies: What are the experts views? En A. M. Teixeira \& A. Szucs (Eds.), Challenges for research into open \& distance learning, conference proceedings eight EDEN research workshop (pp. 27-36). EDEN. https://doi.org/10.38069/edenconf-2014-rw0004

RStudio Team (2020). RStudio: Integrated Development for R. RStudio, PBC, Boston, MA URL http://www.rstudio.com/.

Schön, D. A. (1987). Educating the reflective practitioner: Toward a new design for teaching and learning in the professions. Jossey-Bass.

Sedlacek, M., \& Sedova, K. (2016). How many are talking? The role of collectivity in dialogic teaching. International Journal of Educational Research, 85, 99-108. https://doi.org/10.1016/j.ijer.2017.07.001

Sedova (2017). A case study of a transition to dialogic teaching as a process of gradual change. Teaching and Teacher Education, 67, 278-290.

http://dx.doi.org/10.1016/j.tate.2017.06.018

Sedova, K., Sedlacek, M., Svaricek, R., Majcik, M., Navratilova, J., Drexlerova, A., Kychler, J., Salamounova, Z. (2019). Do those who talk more learn more? The relationship between student classroom talk and student achievement. Learning and Instruction, 63. https://doi.org/10.1016/j.learninstruc.2019.10 1217

Smagorinsky, P., Shelton, S. A., \& Moore, C. (2015). The role of reflexion in developing eupraxis in learning to teach English. Pedagogies: An International Journal, 10(4), 
25-308.

https://doi.org/10.1080/1554480X.2015.1067146

Son, J. B. (2018). Teacher development in technology-enhanced language teaching. Palmgrave Macmillan.

Thorn, C. (2002). Data Use in the Classroom: The Challenges of Implementing Data-based Decision-making at the School Level. University of Wisconsin, Wisconsin Center for Education Research.

Uerz, D., Volman, M., \& Kral, M. (2018). Teacher educators' competences in fostering student teachers' proficiency in teaching and learning with technology: An overview of relevant research literature. Teaching and Teacher Education, 70, 12-23.

https://doi.org/10.1016/j.tate.2017.11.005

van Schaik, P., Volman, M., Admiraal, W. F., \& Schenke, W. (2019). Approaches to coconstruction of knowledge in teacher learning groups. Teaching and Teacher Education, 84, 30-43.

https://doi.org/10.1016/j.tate.2019.04.019

Van Beek, J. A., De Jong, F. P. C. M., Minnaert, A. E. M. G., \& Wubbels, T. (2014). Teacher practice in secondary vocational education: Between teacher-regulated activities of student learning and student selfregulation. Teaching and Teacher Education, 40, 1-9. https://doi.org/10.1016/j.tate.2014.01.005

Vygotsky, L. S. (1978). Mind in society: The development of higher psychological processes. Harvard University Press.

Walsh, S. (2003). Developing interactional awareness in the second language classroom through teacher self-evaluation. Language Awareness, 12(2), 124-142. https://doi.org/10.1080/09658410308667071

Wells, G. (2009). The meaning makers: Learning to talk and talking to learn (2nd edition). Multilingual Matters.

Wong, L., Chai, C. S., \& Aw, G. P. (2017). Seamless language learning: Second language learning with social media. Comunicar, 50, 921. https://doi.org/10.3916/C50-2017-01

Wurth, J. G. R., Tigelaar, E. H., Hulshof, H., de Jong, J. C., \& Admiraal, W. F. (2019). Key elements of L1-oral language teaching and learning in secondary education. A literature review. L1-Educational Studies in Language and Literature, 19, 1-23. https://doi.org/10.17239/L1ESLL2019.19.01.15 